\title{
Impact of fiducial markers placements on the delineation of target volumes in radiation therapy for oesophageal cancer: final results of the FIDUCOR study
}

Laurence Moureau-Zabotto ( $\square$ moureaul@ipc.unicancer.fr)

Institut Paoli-Calmettes https://orcid.org/0000-0002-0341-1812

F. Caillol

Institut Paoli-Calmettes

A. Autret

Institut Paoli-Calmettes

M. Gilabert

Institut Paoli-Calmettes

J. Guiramand

Institut Paoli-Calmettes

J. Darreon

Institut Paoli-Calmettes

M. Ferre

Institut Paoli-Calmettes

$P$ Ries

Institut Paoli-Calmettes

E. Bories

Institut Paoli-Calmettes

M. Tyran

Institut Paoli-Calmettes

A. Tallet

Institut Paoli-Calmettes

M Giovannini

Institut Paoli-Calmettes

Research

Keywords:

Posted Date: April 10th, 2020 
DOI: https://doi.org/10.21203/rs.3.rs-21645/v1

License: (c) (1) This work is licensed under a Creative Commons Attribution 4.0 International License. Read Full License 


\section{Abstract}

Purpose This prospective monocentric phase II study (FIDUCOR-study) aimed at the assessment of the impact of fiducial markers (FMs) implantation on conformal chemo-radiation therapy (CRT) planning in esophageal carcinoma (EC) patients. Methods/materials Fifteen EC patients were enrolled in the study. Each patient underwent two simulation CT-scans before (CT1) and after (CT2) FMs implantation, in the same position. FMs ( $3 \mathrm{~mm}$ length gold markers, preloaded in a $22 \mathrm{G}$ needle) were implanted after sedation, under EUS and X-Ray guidance, and were placed at the tumor's extremities, and in the visible lymph nodes. Target delineation and treatment plan were both performed first on CT1 with the assistance of, diagnosis-CT, gastroscopy- and EUS-details, and second on CT2 using FMs and CT-data. The value of FMs implantation was assessed by the difference of growth-tumor-volume (GTV) and clinical-targetvolume (CTV) between CT1-based and CT2-based delineation. A significant difference was defined as a $\geq 5 \mathrm{~mm}$-difference on axial( $\mathrm{x}$ ) or coronal(y) slices, a $\geq 10 \mathrm{~mm}$-difference on sagittal slices, or $\mathrm{a} \geq 20 \%$ difference in GTV. The impact on dose distribution in organs at risk (OAR) (lung, heart, liver) was also studied. Results Between 09/2014 and 12/2015, 15 patients could achieve fiducial procedures, without any complication. One FM migration occurred. We observed a significant modification of the GTVdimension in $100 \%$ of the cases $(15 / 15,95 \% \mathrm{Cl}$ : [78.2;100.0]), mainly due to a difference in sagittal dimension with a mean variation of $11.2 \mathrm{~mm}$ and a differences $10 \mathrm{~mm}$ for $8 / 15$ patients (53.3\%). One patient had a significant isocenter displacement as high as $20 \mathrm{~mm}$. The esophagus tumor was not seen on the CT-scan in one patient due to its small size. One patient had a distant lymph node metastasis not visible on CT-scan. We observed no significant impact on OAR distribution. Conclusion In our study, FMsimplantation appeared to have positive impact on accurate volume definition in EC-patients. Registration trial number NCT02526134 since the 06-18-2013; URL https://clinicaltrials.gov/ct2/show/NCT02526134

\section{Introduction}

The objective of conformal radiotherapy (CRT) is to improve the dose-distribution, tailored to the target volume borders while reducing the dose to healthy tissues. Accurate delineation of the tumor volume and involved mediastinal lymph nodes is crucial. Computed tomography (CT)-scan is currently the conventional imaging-modality used in Intensity-modulated radiotherapy treatment planning. However, CT does not always allow to distinguish the proximal and distal boundaries between malignant esophageal tumor and healthy esophageal tissues, often because of a poor image-resolution or because the tumor volume can be confused with dietary stasis(Leong et al., 2006). CT is also not well suited to determine mediastinal lymph node involvement. For target volume-definition enhancement, radiotherapists often take advantage of image-registration techniques, mixing various image-exams, especially 18F-fluoro-deoxy-2-d-glucose positron emission tomography (FDG-PET-CT), affording good help in RT-treatment planning and eventually in esophageal carcinoma management(Daisne and Gregoire, 2006;Moureau-Zabotto et al., 2005). Another technique to improve target-definition refers to echoendoscopy (EUS)-assited fiducial markers (FMs) implantation(Pishvaian et al., 2006;Yang et al., 2009; Yang et al., 2011). This technique was shown feasible without major adverse-events in many 
different primary cancers, (i.e., lung, pancreas, prostate cancers(Pishvaian et al., 2006;Yang et al., 2009;Yang et al., 2011), as well as esophagus carcinomas(Jelvehgaran et al., 2017;Jin et al., 2015;Machiels et al., 2015). The impact on the targeting of radiotherapy has been described for the prostatic cancer irradiation(Graf et al., 2010; Yang et al., 2009;Yang et al., 2011). The placement of FMs was also still described for digestive tumors in many lesions such as lymph nodes, esophagus, stomach, pancreas, and biliary tract(Ammar et al., 2010;DiMaio et al., 2010;Jelvehgaran et al., 2017;Jin et al., 2015;Machiels et al., 2015;Park et al., 2010;Pishvaian et al., 2006;Sanders et al., 2010;Varadarajulu et al., 2010). Anyway, the utility of this technique for target volume-definition in radiation therapy (RT) for lesions of the digestive tracts needs investigations. The present study aimed at reporting our findings on the impact of FMs implantation on target volumes-definition in EC-patients considered for definitive or preoperative RT, with or without concurrent chemotherapy. FIDUCOR study, described here, is a nonrandomized, monocentric phase II trial, studying the difference in radiotherapy treatment planning before and after EUS-guided fiducial placement. Anyway, the utility of this technique for target volume-definition in RT for lesions of the digestive tracts needs investigations. The present study aimed at reporting our findings on the impact of FMs implantation on target volumes-definition in EC-patients considered for definitive or preoperative RT, with or without concurrent chemotherapy. FIDUCOR study, described here, is a non-randomized, monocentric phase II trial, studying the difference in radiotherapy treatment planning before and after EUS-guided fiducial placement.

\section{Patients And Methods}

\section{Patient and tumor characteristics}

This single-institution clinical study was conducted between September 2014 and December 2015. Inclusion criteria were as follows: patients more than 18 years old, referred for radiotherapy + /- concurrent chemotherapy for histologically proven esophageal carcinoma (epidermoid or adenocarcinoma). Previously-treated patients for an esophageal tumor were excluded. At referral, the disease was considered to be limited to the esophagus and regional lymph nodes (except in the case of celiac nodal involvement for a distal esophageal tumor and supraclavicular lymph node involvement for an upper esophageal tumor). Patients whom the tumor could not be crossed by the endoscope, and / or had coagulation disorders and /or portal hypertension were excluded from the study. This protocol was approved by an independent ethics committee, and all the patients had to give written informed consent according to all required guidelines (EudraCT number: 2013-A00916-39; NCT02526134).

The pretreatment evaluation included physical examination, complete blood count, biochemistry surveys of liver and kidney function, esophago-gastroscopy with tumor biopsy, chest and abdominal CT-scan, PET-CT-scan, an ear-nose-throat exam for epidermoid tumors, and EUS of the esophagus.

\section{EUS-guided fiducial placement}

In the case of non-distant lymph nodes highlighted by EUS, EUS-guided fiducial placement was performed. The gold linear fiducial markers (EchoTip Ultra-Fiducial-Needle (ETUF), COOK-Medical 
Laboratories) measuring $5 \mathrm{~mm}$ in length $0.64 \mathrm{~mm}$ diameter, were positioned under EUS and X-ray control, in an intubated and supine position patient, using an endoscopic ultrasonography, under general anesthesia. EUS was performed with a slim echoendoscope EG-3270 from Pentax medical. These markers were positioned placed by the gastroenterologist at the superior and the inferior extremities of the tumor, as well as inside the highest and lowest suspect lymph node if a regional lymph node was present.

In the case of distant lymph nodes, a biopsy was performed during the EUS-tumor evaluation. EUS-guided fiducial implantation was performed in a second session, as described above, and a fiducial was also placed in the distant lymph node if proven metastatic on pathological reading.

CT images

Once enrolled in the study, all patients underwent patient underwent two simulation CT-scans (GE optima 580 RT CT-scan) in the same position, in quiet breathing, before (CT1) and after (CT2) FMs implantation. Patients were placed in the supine position using a neck-rest, arms above the head, and lying on a Symmed arm-rest. The alignment was first performed clinically, using the usual anatomic landmarks and three perpendicular laser beams installed in the room. All patients received intravenous injection according to a standard protocol: $110 \mathrm{~mL}$ of non-ionic iodine contrast agent 110 s before image acquisition. . After checking the patient's position on anterior-posterior (A/P) and lateral scoot films, we performed a spiral CT-scan using a 1.5 pitch $2.5 \mathrm{~mm}$ slice thickness, and interslice spacing of $2.5 \mathrm{~mm}$ to encompass the entire thorax and upper abdomen. Four ink-points, corresponding to isocenter and alignment, were tattooed on the patient's chest, at the first simulation-CT time, for positioning purpose.

After FMs implantation, patients underwent the second simulation-CT scan, in the same position using the tattooed marks, and the same window-level settings.

Volumes definition

The Gross Tumor Volume (GTV) was split in GTV-T, corresponding to the esophageal GTV, and GTV-N, corresponding to mediastinal metastatic nodes (defined as nodes with increased FDG uptake or with a short axis of $10 \mathrm{~mm}$ in diameter on CT), (GTV= GTV-T + GTV-N).

Two Clinical Target Volumes (CTV) were defined: (1) the CTV1, encompassing the GTV and a volumetric margin of $4-5 \mathrm{~cm}$ in the cranial-caudal axis, and $1-1.5 \mathrm{~cm}$ in the radial plan limited to anatomical frontiers (lung, heart, sternum, vertebra, big vessels), to account for microscopic tumor extension; (2) the CTV2, encompassing the GTV and a volumetric margin of $2 \mathrm{~cm}$ in the cranial-caudal axis, and $1-1.5 \mathrm{~cm}$ in the radial plan limited to the same anatomical frontiers.

Planning Target volumes (PTV1 and PTV2) were obtained by expanding CTV1 and CTV2, respectively, by a $0.7-1 \mathrm{~cm}$ isotropic margin to account for mean tumor motion. 
All these volumes, as well as Organs At Risk (OARs) (lungs, esophagus, heart, and spinal cord), were delineated on both CT1 and CT2 as described above by the same radiation oncologist to avoid interobserver variability. Target volumes delineation on CT1 was guided by the diagnosis-CT scan, the gastroscopy- and EUS-details, while target volumes delineation on CT2 was FMs and CT-data-guided.

\section{Measures}

We compared GTV from CT1 and CT2 by measuring these volumes using the three dimensions: $\mathrm{X}$ (right left dimension), $Y$ (anterior - posterior dimension), and $Z$ (cranial-caudal dimension) (Figure 1). The largest size of each was the one retained, and the total volumes were also recorded.

Treatment planning was performed according to ICRU Report 83(International Commission on Radiation Units and Measurements, 2017). The dose was prescribed to the ICRU reference point with lung inhomogeneity corrections. The plans were optimized to maximize the PTV dose while lowering the dose to healthy tissue. The PTV was intended to receive a least $95 \%$ and at most $107 \%$ of the prescribed dose to $98 \%$ and $2 \%$ of the PTV, respectively. PTV1 was administered a total dose of 39.6-40 Gy, in 1.8-2 Gydaily fraction, five fractions a week. PTV2 received an additional boost dose with the same fractionation schedule to ensure a mean total dose of 45-60 Gy.

We used the Pinnacle 9.2 treatment planning system (TPS) for all volume delineations and isocenter positioning, then transferred the data to the Ray-station TPS for the plan dose calculation. The calculation of dose distribution was systematically performed for each treatment plan. The cumulative dose/volume histogram was calculated separately for the GTV and OAR, respecting the following constraints (whatever the reference simulation-CT): the maximum radiation dose (Dmax) to the spinal cord did not exceed $45 \mathrm{~Gy}$ (Emami et al., 1991), the maximal percentage of lungs receiving $20 \mathrm{~Gy}$ (V20) was not more than $30 \%$ (Graham et al., 1999). We also compared the following variables: V5 (for the lung), V20 and V30 (for the lung, the liver, and the heart), and V40 (for the heart between the CT1- and CT2-based treatments plans. The Computed Tomography Dose Index for each CT scan was about 13 mGy.cm-1.

Intensity modulated radiation therapy treatments were delivered by a linear accelerator using 6-MV photon beams and using a static (step and shoot) or a dynamic technique .

Statistical analysis

The main objective was the rate of significant change, estimated by the number of observed cases divided by the number of evaluated patients. A 95\% confidence interval was calculated on the estimated proportion. As regards the results of a precedent publication focused on the impact of the TEP-CT on the radiation volumes in esophagus cancer(Moureau-Zabotto et al., 2005), the difference between the two CTscan was considered as significant if one or several of the following criteria was observed:

- difference $\geq 5 \mathrm{~mm}$ in $\mathrm{X}$ (right - left dimension) or $\mathrm{Y}$ (anterior - posterior dimension), 
- difference $\geq 1 \mathrm{~cm}$ in Z (cranial-caudal dimension).

- difference of $>20 \%$ of the GTV

Secondary objectives were: the direction of GTV variation (increased or decreased size) between CT1based and CT2-based delineation, the magnitude of isocenter displacement, the difference in dose received by OARs, and FMs implantation adverse-events. Data were presented by descriptive statistics. Qualitative variables were summarized by frequencies and percentages. Quantitative data were summarized using position (mean, median) and variability (standard deviation, range) statistics.

The statistical analysis was carried out using SAS version 9.3 (SAS Institute, Cary, NC, USA).

\section{Results}

FMs implantation procedure:

Fifteen EC-patients were enrolled in the study and could achieve fiducial implantation procedures. At least one FM was successfully implanted both at the upper and lower limits of the tumor (range: 1-2, in the two locations) in all patients, while 0 to $4 \mathrm{FM}$ (median:1) were implanted in suspicious lymph nodes (Fig. 2). No complication occurred during and after the procedure. For one patient, we observed the migration of one FM.

Impact on Target volume delineation

The mean GTV volume was $57.3 \mathrm{~cm} 3$ (median: 50.7; range: [5.0-152.4]) without FMs and $77.0 \mathrm{~cm} 3$ (median: 60.2; range: [8.7-225.7]) with FMs. Details of GTV modifications for each patient are given in table 1.

In one patient, the GTV (GTV-T + GTV-N) significantly decreased with the use of FMs, with a mean decrease of $26.4 \mathrm{~cm} 3$, whereas for ten patients, the use of FMs for delineation significantly increased the GTV, with a mean increase of $71.4 \mathrm{~cm} 3$ (median: 57.4; range: [24.7-175.1]). In one patient, the GTV increased because the FMs placement allowed the discovery of an occult positive node in the mediastinum. In another patient, with a T1NO lesion, the tumor, too small to be visible on CT1, was detected by EUS, and FMs implantation allowed its correct delineation. A $2 \mathrm{~cm}$ - displacement of the isocenter in the cranial-caudal dimension was observed in one patient. Overall, the GTV was significantly modified by the use of FMs in the cranial-caudal dimension, with a mean difference of $28.9 \mathrm{~mm}$ (standard deviation: 21.9) between the two CT-scan. The mean length of the GTV (including macroscopic nodes) without FMs use was $72.9 \mathrm{~mm}$ (median: 70.0; range: [27.5-166.2]) vs. $94.5 \mathrm{~mm}$ with FMs use (median: 85.0; range: [42.5-142.5]).

The mean length of the esophageal tumor (GTV-T) without FMs use was $61.0 \mathrm{~mm}$ (median: 57.5; range: [27.5-112.5]) vs. $69.2 \mathrm{~mm}$ with FMs use (median: 65.0; range: [30.0-140.0]). A reduction of the esophageal tumor length was observed in 3 patients (mean: $31.4 \mathrm{~mm}$, median: 17.0, range:[12.5-64.7]). 
An esophageal tumor-length increase was observed in 6 patients (mean increase: $30.7 \mathrm{~mm}$, median:27.0, range: $16.5-63.2)$.

Impact on the total dose delivered to the organ at risk

The mean percentage of lung receiving $5 \mathrm{~Gy}$ (V5), $20 \mathrm{~Gy}$ (V20) and $30 \mathrm{~Gy}$ (V30) was 67.7\% (range: [31.297.5]), 16.5\% (range: [2.0-30.7]) and 5.8\% (range: [0.74-11.9]), respectively, before FMs placement versus 66.8\% (range: [28.8-91.8]), 18.0\% (range: [3.1-34.0]), and 5.9\% (range: [0.9-14.1]), respectively, after FMs placement. The mean dose (Dmean) delivered to the lungs was $11.8 \mathrm{~Gy}$ (range: [4.2-17.2]) before FMs placement versus 11.7 Gy (range: [4.5-16.4]) after FMs placement.

The mean percentage of heart receiving 20 Gy (V20), 30 Gy (V30) and 40 Gy (V40) was $41.4 \%$ (range: [20.3-85.0]), 20.0\% (range: [7.6-43.7]) and 10.3\% (range: [3.3-22.5]), respectively, before FMs placement versus $40.7 \%$ (range: [25.8-65.0]), 18.6\% (range: [11.2-30.2]), and 8.9\% (range: [0.7-19.6]), respectively, after FMs placement. The mean of the mean and the maximum dose delivered to the heart was respectively $20.3 \mathrm{~Gy}$ (range: [12.3-31.1]) and 49.4 Gy (range: [45.6-61.6]) before FMs placement versus respectively 19.7 Gy (range: (15.4-26.3]) and 48.7 Gy (range: [39.8-59.7]) after FMs placement.

The mean percentages of liver receiving 20 Gy (V20) and 30 Gy (V30) were 24.8\% (range: [0.0-44.9]), and $15.5 \%$ (range: [0.0-77.7]), respectively, before FMs placement versus $28.9 \%$ (range: [0.0-56.1]), and $11.6 \%$ (range: [0.0-22.8]), respectively, after FMs placement. The mean dose delivered to the liver was $13.3 \mathrm{~Gy}$ (range: [0.2-20.4]) before FMs placement versus $13.7 \mathrm{~Gy}$ (range: (0.7-21.7]) after FMs placement.

\section{Discussion}

An optimal dose delivery, avoiding geographic misses, and reducing the volume of healthy tissue irradiated, would ensure maximal tumor local control, without compromising the quality of life. Current standard practice in EC volume definition, in the setting of curative intent external beam CRT, relies on the combination of CT and PET-CT datasets(Crehange et al., 2016;Fraass, 1995; Vrieze et al., 2004). FDG-PET success in identifying most primary tumors, with a 30-93\%(Kole et al., 1998;Yoon et al., 2003) sensitivity and a 79-100\%(Sihvo et al., 2004) specificity for the detection of metastatic lymph nodes. In a prospective study comparing PET-scan, CT-scan and ultrasonography in the diagnosis of esophageal and esophagogastric junction cancers, was shown of limited value due to a weak accuracy in para-tumoral and distant lymph nodes staging of. Although PET-scan was proven superior to CT-scan in metastases detection(Rasanen et al., 2003), low evidence supports its use in tumor delineation, mainly explained by the inflammation surrounding the tumor, leading to false-positive uptakes(Metser and Even-Sapir, 2007).

On the other hand, the use of FMs to enhance RT-volume definition is actually validated and routinely used in prostate cancer irradiation(Graf et al., 2010;Yang et al., 2009;Yang et al., 2011). Fiducials implant was also still described in many digestive tumors such as lymph nodes, esophagus, stomach, pancreas, and biliary tract, but never used to guide radiotherapy(Ammar et al., 2010;DiMaio et al., 2010;Jelvehgaran et al., 2017; Jin et al., 2015;Machiels et al., 2015;Park et al., 2010;Pishvaian et al., 2006;Sanders et al., 
2010;Varadarajulu et al., 2010). Endoscopic placement of fiducial markers for radiation therapy guidance is a relatively newer application of EUS in pancreatic and thoracic tumors. One recent report described the successful use of fiducials placed under linear EUS guidance only in patients with abdominal and mediastinal tumors, but this report did not address EC-patients(Pishvaian et al., 2006).

Our study is a prospective monocentric study, conducted in a short period, avoiding the potential bias of inhomogeneity between different teams for the placement of FM. Furthermore, target volume delineation was done by the same radiotherapist, both before and after FM placement, avoiding inter-observer delineation variability. Moreover, the radiotherapist strictly respected the rules to measure the largest dimension for each tumor, as well as the distances from the superior and the inferior extremities of the FM to reduce the risk of intra-operator and inter-operator variability (Figure 1). Computed Tomography Dose Index (CTDI) for each CT scan was $13 \mathrm{mGy} . \mathrm{cm}-1$. So, for a $50 \mathrm{~cm}$ CT length, which is a common length for a RT CT simulation, the extra dose was $0.6 \mathrm{~Gy}$ for each patient enrolled in the study. Related to the total dose delivered, CT extra dose could be considered as negligible. In addition, the 2 CT scans (before and after FM placement) were fused together, with a rigid registration, focusing on vertebrates. Time between CT scans, 15 days maximum, was short enough not to observe significant anatomical changes. Uncertainties were evaluated for each case and considered as negligible.

In this study, and according to our main objective criteria (composite parameter), we observed that FM implantation significantly modified the GTV in $15 / 15$ patients (100\%, Cl 95\%: [78.2-100.0]), mainly due to an increase in this volume (10/15, 66.7\%). A GTV modification was mainly observed in the cranial-caudal dimension, being statistically significant in $8 / 15$ patients (53.3\%). FMs implantation also led to the discovery of occult lymphadenopathy in one patient, EUS being already well-described in the literature as an efficient tool for occult-distant lesions detection(Ammar et al., 2010;Araujo et al., 2013;Mortensen et al., 2001). More interesting was the case of one patient medically unfit for surgery and harboring a too small lesion to be visible on both the CT and the PET-CT-scans (only visualized by endoscopy): in whom only FMs placement made target volume definition possible.

Whereas FMs-driven volume modification appears of lower magnitude in PTV and has little impact on OARs dose distribution, the increasing use of hypofractionated radiotherapy schedules, with higher dosefractionation and lower margins around the GTV, makes FMs implantation an attractive method for the accurate definition of RT-target volumes.

However, our study presents some limitations. First and the small sample size requires confirmation of the findings through a prospective study, currently ongoing in France (FIDECHO). Second, this technique is feasible only in the case of tumors that can be easily crossed by endoscopy. We used in this study a slim echo-endoscope (EG-3270UK from Pentax medical ${ }^{\circledR}$ ), thinner than usual echo-endoscopes, which allowed to cross all the esophagus tumors. Noteworthy, fiducials also had the advantage of their radioopacity, lowering patient setup errors during the treatment course and also reducing healthy tissue (pulmonary, cardiac and esophageal) irradiation. However, the aim of our study was not to demonstrate such an interest, and this potential advantage might be investigated in specific study. 
To our knowledge, this is the first study to demonstrate the potential interest of this technique in the definition of RT-target volume in digestive tract lesions, especially in esophagus radiotherapy. These data will be expanded by the ongoing larger scale prospective French multicenter FIDECHO study.

\section{Conclusion}

This study suggested a positive-impact of FM the implantation for the definition of RT-target volumes in esophagus radiotherapy. A larger scale, prospective multicenter study is currently underway to validate our preliminary data.

\section{Abbreviations}

- fiducial markers (FMs)

- chemo-radiation therapy (CRT)

- esophageal carcinoma (EC)

- CT-scan before (CT1) and after (CT2) FMs implantation,

- Endoscopic Ultrasound : EUS

- growth-tumor-volume (GTV)

- clinical-target-volume (CTV)

- Planning Target volume (PTV)

- organs at risk (OAR)

- Gray (Gy)

- 18F-fluoro-deoxy-2-d-glucose positron emission tomography (FDG-PET-CT)

- radiation therapy $(\mathrm{RT})$

- EchoTip Ultra-Fiducial-Needle (ETUF)

\section{Declarations}

Ethics approval and consent to participate:

This protocol was approved by an independent ethics committee (cpp Sud Méditerranéen, réf 1373) and all the patients had to give written informed consent according to all required guidelines (EudraCT number: 2013-A00916-39; NCT02526134). 
Consent for publication : Not applicable

Availability of data and materials: Please contact author for data requests.

Funding: The authors have no source of funding for the research to declare

Competing interests: The authors declare that they have no competing interests.

Authors' contributions :

MZL, CF, CS have been involved in the conception and the design of the study. MZL, CF, GM, GJ, BE, TM, TA, GM contributed to the provision of study materials or patients. MZL, CF, CS, AA, DJ, FM proceed to the collection and assembly of data. Data analysis and interpretation was realized by MZL, CF, CS and AA. MZL, CF, DJ, FM wrote the manuscript. All authors read and approved the final manuscript

Acknowledgements Not applicable

\section{References}

Ammar, T., G. A. Cote, K. M. Creach, C. Kohlmeier, P. J. Parikh, and R. R. Azar, 2010, Fiducial placement for stereotactic radiation by using EUS: feasibility when using a marker compatible with a standard 22-gauge needle: Gastrointest Endosc, v. 71, no. 3, p. 630-633.

Araujo, J. et al., 2013, Distant lymph node metastases in gastroesophageal junction adenocarcinoma: impact of endoscopic ultrasound-guided fine-needle aspiration: Endosc Ultrasound, v. 2, no. 3, p. 148-152.

Crehange, G., F. Huguet, L. Quero, T. V. N'Guyen, X. Mirabel, and T. Lacornerie, 2016, [Radiotherapy in cancers of the oesophagus, the gastric cardia and the stomach]: Cancer Radiother, v. 20 Suppl, p. S161S168.

Daisne, J. F., and V. Gregoire, 2006, Imagerie multimodale pour la définition des volumes cibles en radiothérapie: Bull Cancer, v. 93, no. 12, p. 1175-1182.

DiMaio, C. J., S. Nagula, K. A. Goodman, A. Y. Ho, A. J. Markowitz, M. A. Schattner, and H. Gerdes, 2010, EUS-guided fiducial placement for image-guided radiation therapy in GI malignancies by using a 22gauge needle (with videos): Gastrointest Endosc, v. 71, no. 7, p. 1204-1210.

Emami, B., J. Lyman, A. Brown, L. Coia, M. Goitein, J. E. Munzenrider, B. Shank, L. J. Solin, and M. Wesson, 1991, Tolerance of normal tissue to therapeutic irradiation: Int J Radiat Oncol Biol Phys, v. 21, no. 1, p. 109-122.

Fraass, B. A., 1995, The development of conformal radiation therapy: Med Phys, v. 22, no. 11 Pt 2, p. 1911-1921. 
Graf, R., D. Boehmer, V. Budach, and P. Wust, 2010, Residual translational and rotational errors after kV Xray image-guided correction of prostate location using implanted fiducials: Strahlenther.Onkol., v. 186, no. 10, p. 544-550.

Graham, M. V., J. A. Purdy, B. Emami, W. Harms, W. Bosch, M. A. Lockett, and C. A. Perez, 1999, Clinical dose-volume histogram analysis for pneumonitis after 3D treatment for non-small cell lung cancer (NSCLC): Int J Radiat Oncol Biol Phys, v. 45, no. 2, p. 323-329.

International Commission on Radiation Units and Measurements. ICRU Report 83: Prescribing, Recording, and Reporting Intensity-Modulated Photon-Beam Therapy (IMRT). 2017. Bethesda. 2017.

Ref Type: Report

Jelvehgaran, P., T. Alderliesten, J. J. A. Weda, B. M. de, D. J. Faber, M. C. C. M. Hulshof, T. G. van Leeuwen, H. M. van, and J. F. de Boer, 2017, Visibility of fiducial markers used for image-guided radiation therapy on optical coherence tomography for registration with CT: An esophageal phantom study: Med Phys, v. 44 , no. 12 , p. 6570-6582.

Jin, P. et al., 2015, Marker-based quantification of interfractional tumor position variation and the use of markers for setup verification in radiation therapy for esophageal cancer: Radiother Oncol, v. 117, no. 3, p. 412-418.

Kole, A. C., J. T. Plukker, O. E. Nieweg, and W. Vaalburg, 1998, Positron emission tomography for staging of oesophageal and gastroesophageal malignancy: Br J Cancer, v. 78, no. 4, p. 521-527.

Leong, T. et al., 2006, A prospective study to evaluate the impact of FDG-PET on CT-based radiotherapy treatment planning for oesophageal cancer: Radiother Oncol, v. 78, no. 3, p. 254-261.

Machiels, M., H. J. van, P. Jin, M. I. van Berge Henegouwen, H. M. van Laarhoven, T. Alderliesten, and M. C. Hulshof, 2015, Endoscopy/EUS-guided fiducial marker placement in patients with esophageal cancer: a comparative analysis of 3 types of markers: Gastrointest Endosc, v. 82, no. 4, p. 641-649.

Metser, U., and E. Even-Sapir, 2007, Increased (18)F-fluorodeoxyglucose uptake in benign, nonphysiologic lesions found on whole-body positron emission tomography/computed tomography (PET/CT): accumulated data from four years of experience with PET/CT: Semin Nucl Med, v. 37, no. 3, p. 206-222.

Mortensen, M. B., T. Pless, J. Durup, A. P. Ainsworth, G. J. Plagborg, and C. Hovendal, 2001, Clinical impact of endoscopic ultrasound-guided fine needle aspiration biopsy in patients with upper gastrointestinal tract malignancies. A prospective study: Endoscopy, v. 33, no. 6, p. 478-483.

Moureau-Zabotto, L. et al., 2005, Impact of CT and 18F-deoxyglucose positron emission tomography image fusion for conformal radiotherapy in esophageal carcinoma: Int J Radiat Oncol Biol Phys, v. 63, no. 2, p. 340-345. 
Park, W. G., B. M. Yan, D. Schellenberg, J. Kim, D. T. Chang, A. Koong, C. Patalano, and D. J. Van, 2010, EUS-guided gold fiducial insertion for image-guided radiation therapy of pancreatic cancer: 50 successful cases without fluoroscopy: Gastrointest Endosc, v. 71, no. 3, p. 513-518.

Pishvaian, A. C., B. Collins, G. Gagnon, S. Ahlawat, and N. G. Haddad, 2006, EUS-guided fiducial placement for CyberKnife radiotherapy of mediastinal and abdominal malignancies: Gastrointest Endosc, v. 64 , no. 3, p. $412-417$.

Rasanen, J. V., E. I. Sihvo, M. J. Knuuti, H. R. Minn, M. E. Luostarinen, P. Laippala, T. Viljanen, and J. A. Salo, 2003, Prospective analysis of accuracy of positron emission tomography, computed tomography, and endoscopic ultrasonography in staging of adenocarcinoma of the esophagus and the esophagogastric junction: Ann Surg Oncol, v. 10, no. 8, p. 954-960.

Sanders, M. K., A. J. Moser, A. Khalid, K. E. Fasanella, H. J. Zeh, S. Burton, and K. McGrath, 2010, EUSguided fiducial placement for stereotactic body radiotherapy in locally advanced and recurrent pancreatic cancer: Gastrointest Endosc, v. 71, no. 7, p. 1178-1184.

Sihvo, E. I., J. V. Rasanen, M. J. Knuuti, H. R. Minn, M. E. Luostarinen, T. Viljanen, M. A. Farkkila, and J. A. Salo, 2004, Adenocarcinoma of the esophagus and the esophagogastric junction: positron emission tomography improves staging and prediction of survival in distant but not in locoregional disease: $\mathrm{J}$ Gastrointest Surg, v. 8, no. 8, p. 988-996.

Varadarajulu, S., J. M. Trevino, S. Shen, and R. Jacob, 2010, The use of endoscopic ultrasound-guided gold markers in image-guided radiation therapy of pancreatic cancers: a case series: Endoscopy, v. 42, no. 5, p. 423-425.

Vrieze, O., K. Haustermans, W. W. De, T. Lerut, C. E. Van, N. Ectors, M. Hiele, and P. Flamen, 2004, Is there a role for FGD-PET in radiotherapy planning in esophageal carcinoma?: Radiother Oncol, v. 73, no. 3, p. 269275 .

Yang, J., M. Abdel-Wahab, and A. Ribeiro, 2009, EUS-guided fiducial placement before targeted radiation therapy for prostate cancer: Gastrointest Endosc, v. 70, no. 3, p. 579-583.

Yang, J., M. Abdel-Wahab, and A. Ribeiro, 2011, EUS-guided fiducial placement after radical prostatectomy before targeted radiation therapy for prostate cancer recurrence: Gastrointest Endosc, v. 73, no. 6, p. 1302-1305.

Yoon, Y. C., K. S. Lee, Y. M. Shim, B. T. Kim, K. Kim, and T. S. Kim, 2003, Metastasis to regional lymph nodes in patients with esophageal squamous cell carcinoma: CT versus FDG PET for presurgical detection prospective study: Radiology, v. 227, no. 3, p. 764-770.

\section{Tables}


Table 1:

\begin{tabular}{|c|c|c|c|c|c|}
\hline \multirow[b]{2}{*}{ Patient } & \multicolumn{3}{|c|}{$\begin{array}{l}\text { GTV dimensions before/after FM : difference (increase } \\
(+) \text { or decrease }(-)(\mathrm{mm})\end{array}$} & \multirow{2}{*}{$\begin{array}{l}\text { GTV before/after FM (cc): difference (increase (+) } \\
\text { or decrease (-), \%) } \\
\text { volume }\end{array}$} & \multirow{2}{*}{$\begin{array}{l}\text { Significant } \\
\text { yes / no } \\
\text { (significant axis) }\end{array}$} \\
\hline & $\begin{array}{l}\text { Left-Right } \\
\text { (x) }\end{array}$ & $\begin{array}{l}\text { Anterior-Posterior } \\
\text { (y) : }\end{array}$ & $\begin{array}{l}\text { Superior-inferior } \\
\text { (z) }\end{array}$ & & \\
\hline 1 & 41/41: 0 & 33/34: +1 & 104/87: -17 & $72 / 60: \quad-17 \%$ & Yes (z) \\
\hline 2 & $30 / 24:-6$ & 23/19: -4 & $73 / 83:+10$ & 20/21: $\quad+5 \%$ & Yes $(\mathrm{x}, \mathrm{z})$ \\
\hline 3 & $\begin{array}{l}36 / 40.5: \\
+4.5\end{array}$ & $22 / 24:+2$ & 139/130: -9 & 16/44: $+175 \%$ & Yes (volume) \\
\hline 4 & $43 / 46: \quad+3$ & 41/32: -9 & 45/88: +43 & 38/43: $+16 \%$ & Yes (z) \\
\hline 5 & 44/40: -4 & 60/60: 0 & $38 / 14:-24$ & 49/66: $+35 \%$ & Yes (z,volume) \\
\hline 6 & 19/19: 0 & 14/21: +7 & $25 / 52:+27$ & 50/87: $+74 \%$ & Yes (y, z,volume) \\
\hline 7 & 69/62: -7 & 60/65: +5 & 98/96: -2 & 141/140: $-0.7 \%$ & Yes $(x, y)$ \\
\hline 8 & $53 / 58:+5$ & 40/61: +21 & 56/44: -12 & 51/119: $133 \%$ & $\begin{array}{l}\text { Yes }(\mathrm{x}, \mathrm{y} \\
\mathrm{z}, \mathrm{volume})\end{array}$ \\
\hline 9 & $31 / 25:-6$ & 26/36: +10 & $36 / 59:+23$ & $23 / 34: \quad+48 \%$ & $\begin{array}{l}\text { Yes }(\mathrm{x}, \mathrm{y} \\
\mathrm{z} \text {,volume })\end{array}$ \\
\hline 10 & $39 / 41:+2$ & 27/25: -2 & $75 / 145:+70$ & $36 / 60: \quad+67 \%$ & Yes (z,volume) \\
\hline 11 & 32/31:-1 & 28/24:-4 & 63/60: -3 & 51/38: $\quad-25 \%$ & Yes (volume) \\
\hline 12 & 52/52: 0 & 37/37: 0 & 57/74: +16 & 86/109: $+27 \%$ & Yes (z,volume) \\
\hline 13 & $38 / 42:+4$ & $43 / 39:-4$ & $51 / 115:+64$ & 67/123: +81\% & Yes (z,volume) \\
\hline 14 & 40/40:0 & 25/24:-1 & 106/136: +30 & $52 / 65: \quad+25 \%$ & Yes (z,volume) \\
\hline 15 & 49/64:+15 & 45/48: +3 & $117 / 144:+27$ & 152/225: +48\% & Yes (x, z,volume) \\
\hline
\end{tabular}

Figures

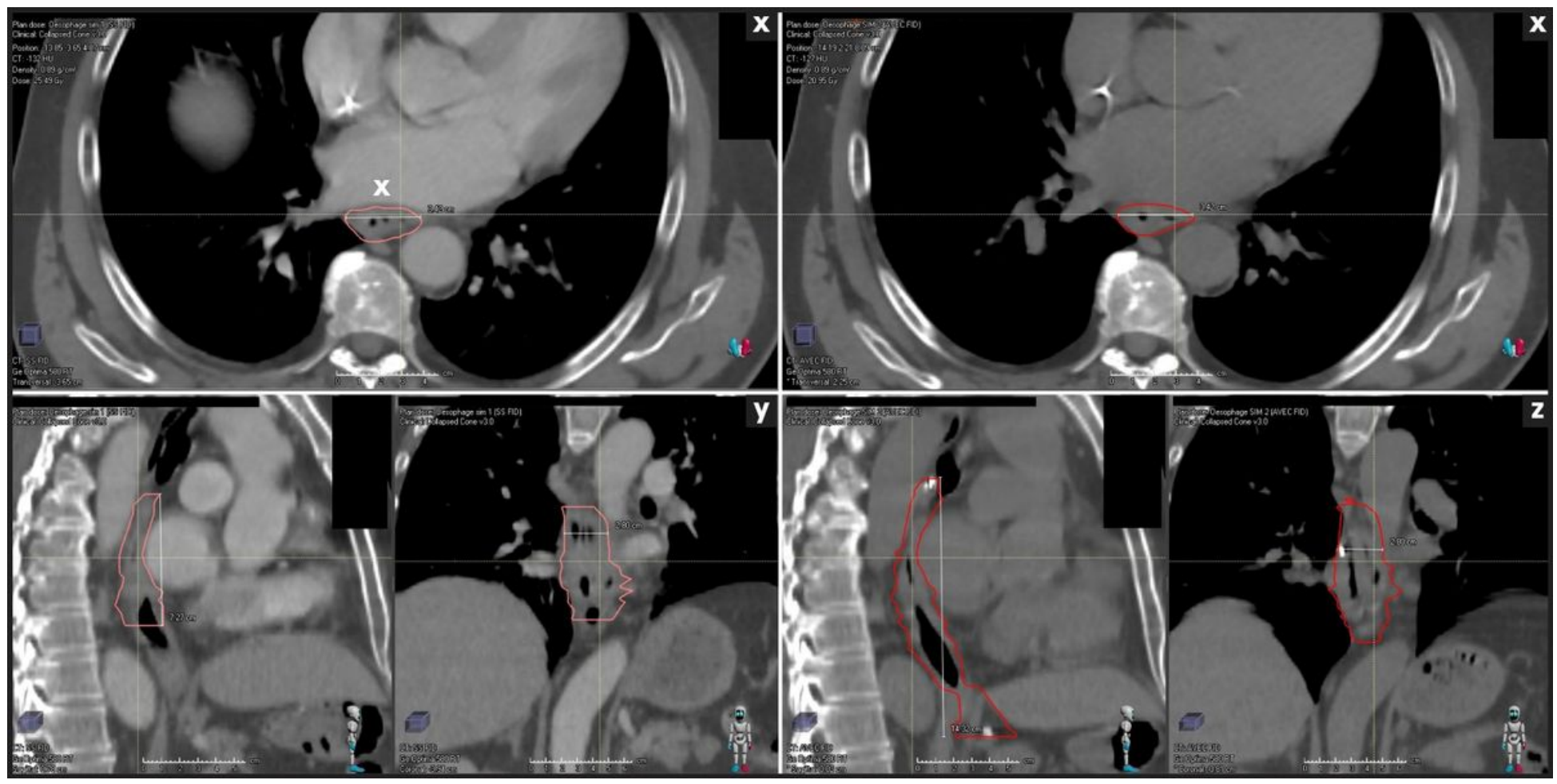


Figure 1

Example of measurement of GTV X (right - left dimension) (a) or Y (anterior - posterior dimension)(b) , or Z (cranio-caudal dimension) (c).

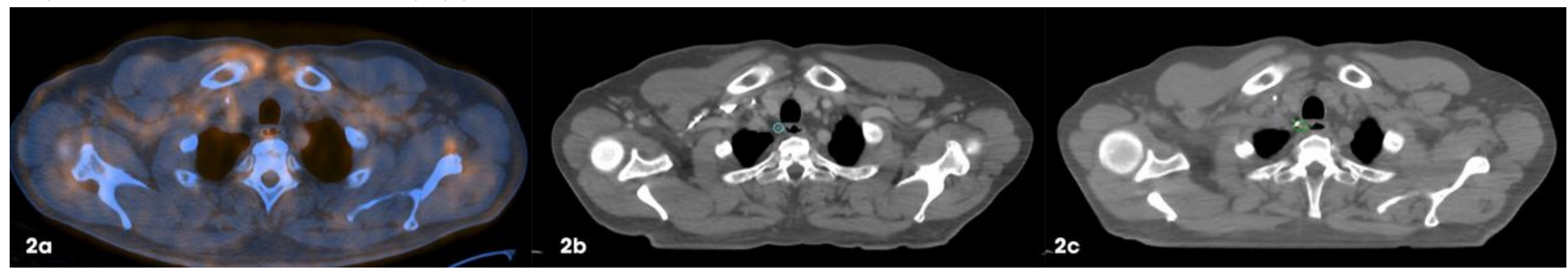

Figure 2

Example of a lump node on the TEP-CT (a) , and on the two simulation CT-scans : before(b) and after FM placement (c) 